ben“ (S. 28). Diese Bedingung außenpolitischen Handelns beschreibt jedoch keineswegs ein Novum, sondern vielmehr ein etabliertes Merkmal deutscher Außenpolitik. Gerade durch den Verzicht einer nationalstaatlich auftrumpfenden Politik hat die Bundesrepublik schon in ihrer Anfangszeit Respekt und Einflussmöglichkeiten hinzugewonnen. Als Motor des europäischen Einigungswerkes sowie durch multilaterale Einbindung ist das deutsche Regierungssystem mittlerweile so weit gezähmt, dass eine rein national gefärbte Politik kaum mehr vorstellbar ist. In seinem Beitrag zur „Demokratie in Deutschland“ konstatiert Dietrich Thränhardt, dass der Nationalstaat in Deutschland lange Zeit keine dominante Leitkategorie gewesen sei und dies zu einer „Scheu vor Staatssymbolen“ (S. 146) geführt habe. So sei auch zu erklären, warum die Ereignisse von 1989/90 nicht als „friedliche Revolution“, sondern als „Wende“ bezeichnet werden. Dieser Zusammenhang bleibt allerdings fraglich, handelt es sich doch vor allem um einen Wahrnehmungsunterschied zwischen Ost und West. Während die Bürger in Ostdeutschland mehrheitlich von Wende sprechen, hat sich bei den Westdeutschen eher das Wort von der Wiedervereinigung durchgesetzt.

Insgesamt fällt auf, dass das Handwörterbuch eine starke Polity-Orientierung aufweist. Institutionen und Politikfelder werden durch unterschiedliche Stichworteinträge abgedeckt, Mechanismen und politische Verfahren bleiben jedoch etwas unterrepräsentiert. Zwar gibt es Einträge zu „Politische Beteiligung“, „Direkte Demokratie“ oder „Pluralismus“. Zentrale Konzepte wie „Politische Steuerung“ oder „Politikverflechtung“ sind hingegen nicht dabei. Für das Verständnis des politischen Systems und seiner Problemlösungsverfahren könnten diese Stichwörter ganz sicher zusätzliche Erkenntnisse liefern. Abgesehen von diesen einschränkenden Befunden handelt es sich bei dem vorliegenden Band um ein nützliches Nachschlagewerk, das man für eine erste Orientierung oder einfach nur noch einmal zur eigenen Wissensauffrischung gut zu Rate ziehen kann. Werden die erwähnten Schwachpunkte in der nächsten Auflage behoben, kann das Handwörterbuch noch ein Stück an Qualität hinzugewinnen.

Helge F. Jani

\title{
Populäre Aufbereitungen des Deutschen Bundestages
}

Das deutsche Parlament. Mit Beiträgen von Manfred Görtemaker, Everhard Holtmann, Wolfgang Ismayr, Michael S. Cullen und Volker Wagner, Verlag Barbara Budrich, Opladen \& Farmington Hills 2009, 272 Seiten, € 39,90.

Feldkamp, Michael F: Der Deutsche Bundestag. 100 Fragen und Antworten, Nomos Verlagsgesellschaft, Baden-Baden 2009, 208 Seiten, € 19,90.

Im September 2009 feierte der Bundestag den 60. Jahrestag seines Bestehens. Im Gegensatz zum Grundgesetz, das im Mai 60 Jahre alt geworden war, stand der Bundestag allerdings nicht im publizistischen Fokus. Während zur deutschen Verfassung mehrere Bücher veröffentlicht wurden (sogar eines für Kinder) ${ }^{1}$, liegt zum Parlament eine solche Anzahl an

1 Christian Bommarius, Das Grundgesetz. Eine Biographie, Berlin 2009; Christoph Möllers, Das Grundgesetz. Geschichte und Inhalt, München 2009; Marion Detjen / Stephan Detjen / 
Publikationen nicht vor. Die letzten an eine größere Leserschaft adressierten stammen aus dem Jahr 2007 und waren eher didaktisch ausgerichtet. ${ }^{2}$ Immerhin sind im letzten Jahr zwei Werke zum Bundestag erschienen, die sich an ein breites Publikum wenden: ein Bildband mit fünf Texten sowie ein Fragen- und Antwortenbuch.

Das reich bebilderte und nunmehr zum fünften Mal aufgelegte Buch „Das deutsche Parlament" hat sich gegenüber seinen vier Vorgängern verändert. Letztere enthielten jeweils drei Beiträge von Wolfgang Gaebler, Wolfgang Kessel und Hans-Achim Roll, deren englische und französische Übersetzungen in Spalten neben der deutschen Fassung angeordnet waren. Der Bundestag fungierte als Herausgeber, und man kann sich gut vorstellen, dass diese großformatigen Bücher gern von der Institution als Geschenk überreicht wurden. Die erste Auflage erschien 1989 unter Bundestagspräsidentin Rita Süssmuth, die weiteren 1990, 1994 und 1999, jeweils im Kohlhammer Verlag. Die fünfte Auflage ist nun ohne Herausgeberschaft des Parlaments und in einem neuen Verlag publiziert worden. Es wurden drei neue Autoren gewonnen, die die drei hergebrachten Themen zur Geschichte des Parlamentarismus in Deutschland bis 1945, die Zeit bis zur Vereinigung 1990 und den Bundestag als Institution bearbeiten. Hinzu gekommen sind zwei Texte zum Reichstagsgebäude und zum Parlamentsviertel. Die Beiträge sind jetzt nur noch auf deutsch abgedruckt. Eine komplett englische Fassung des Buches ist separat erschienen. ${ }^{3}$

Manfred Görtemaker schaut zurück auf die Entwicklung des Parlamentarismus in Deutschland, beginnend mit den preußischen Reformbestrebungen Anfang des 19. Jahrhunderts über die Paulskirchenversammlung von 1848, den Reichstag der Kaiserzeit und dann der Weimarer Republik bis hin zur faktischen Nichtexistenz jeglichen Parlamentarismus von 1933 bis 1945. Everhard Holtmann knüpft hier an und blickt auf die Entstehung des Grundgesetzes. Danach geht er - im Gegensatz zu seinem Vorgängerbeitrag aus den ersten vier Auflagen - weniger chronologisch vor, sondern befasst sich auch mit Grundfragen des deutschen politischen Systems wie der Kanzlerdemokratie, dem kooperativen Föderalismus oder der These vom Funktionsverlust des Parlaments. Daneben lässt er entscheidende Daten der bundesrepublikanischen Geschichte Revue passieren bis hin zur deutschen Vereinigung 1990 und ihren Auswirkungen. Den Bundestag als Institution behandelt Wolfgang Ismayr. Er thematisiert das Wirken der Abgeordneten, die arbeitsteilige Gliederung des Bundestags in Fraktionen und Ausschüsse sowie die Funktionen des Parlaments. Auf seine bisherigen Veröffentlichungen baut Michael S. Cullen auf und liefert einen kurzen Abriss zur Geschichte des Reichstagsgebäudes, wobei er auch den Weg zur Verhüllung des Reichstags 1995 nachzeichnet. Abschließend wandert Volker Wagner durchs Parlamentsviertel, erläutert die architektonischen Besonderheiten des Reichstags und der drei Parlamentsneubauten und widmet sich den vornehmlich modernen Kunstobjekten in diesen vier Gebäuden.

Maximilian Steinbeis, Die Deutschen und das Grundgesetz. Geschichte und Grenzen unserer Verfassung, München 2009; Peter Zolling, Das Grundgesetz. Unsere Verfassung - wie sie entstand und was sie ist, München 2009; Karen-Susan Fessel / Manfred Schwarz, GG - Was ist das? Das Grundgesetz erklärt, Hamburg 2009.

2 Uwe Andersen (Hrsg.), Der Deutsche Bundestag, Schwalbach am Taunus 2007; Bundeszentrale für politische Bildung (Hrsg.), Parlamentarische Demokratie, Informationen zur politischen Bildung Nr. 295, Bonn 2007.

3 The German Parliament, Verlag Barbara Budrich, Opladen / Farmington Hills 2009. 
Die fünf Beiträge sind durchweg nüchtern-sachlich im Ton gehalten. Sie erlauben interessierten Lesern einen Einblick in den Bundestag, seine Geschichte, seine Vorläufer und seine Architektur. Durch die vielen Abbildungen regt das Buch zudem zum Durchblättern an. Es ist auch in seiner Aufmachung ein repräsentativer Band, der sich aufgrund der Auswahl an zeitgenössischen Photos und der soliden Grundlagentexte durchaus auch für einen fortgeschrittenen Kenner des Parlaments eignet. So findet sich hier zum Beispiel eine Reproduktion des Intarsienbildes aus dem alten Reichstag, auf das, wenn es um die Entstehung der Bezeichnung Hammelsprung geht, gern verwiesen wird (S. 207).

$\mathrm{Zu}$ fragen bleibt allerdings, ob eine Publikation mit dem Titel „Das deutsche Parlament“ nicht stärker auf Entwicklungen in den Landesparlamenten hätte eingehen können und legt man ein weites Parlamentsverständnis zugrunde - einen Blick auf die Volkskammer der DDR zwischen 1949 und 1989 hätte werfen sollen. Auch Quellenangaben finden sich leider nicht. Das ist zwar erklärbar, da die Publikation ein größeres Publikum zu interessieren hofft, aber auch solche Leser möchten vielleicht dieses oder jenes noch einmal nachschlagen oder eine Anregung zum Weiterlesen aufnehmen. Zumindest ein kleiner Anmerkungsapparat im Anhang hätte hier Abhilfe schaffen können. Bedauerlich ist zudem, dass sich einige Fehler in Bildunterschriften eingeschlichen haben. So werden Wilhelm Pieck und Otto Grotewohl verwechselt (S. 62), die Berliner Mauer stand gleich 40 Jahre (S. 79), und die am 18. März 1990 gewählte Volkskammer fand sich demnach noch am Wahltag zu ihrer konstituierenden Sitzung zusammen (S. 115).

Erhebt „Das deutsche Parlament“ den Anspruch, Wissenswertes über den Bundestag zugänglich aufzubereiten, versammelt Michael F. Feldkamp in seinem Taschenbuch „Der Deutsche Bundestag“, wie er selbst im Vorwort schreibt, auch „(N)icht-(W)issenswertes“ (S. 5). Hervorgegangen sind die „100 Fragen und Antworten“ (so der Untertitel) aus einem Sudelbuch, in dem Feldkamp, der in der Verwaltung des Bundestages tätig ist, Notizen seines Arbeitsalltags festgehalten hat. Er bietet ein breites Spektrum an Themen: Parlamentsgeschichte, Wahlen, Fraktionen, Abgeordnete, Präsidium, Plenargeschehen, Gesetzgebung, Kontrolle, Gebäude und Verwaltung. Die Fragen sind dabei recht unterschiedlicher Natur: Mal geht es um statistische Informationen wie Wahlergebnisse und Mandatsverteilungen, mal um Wissensfragen (Was bedeutet allgemeine, unmittelbare, freie, gleiche und geheime Wahl?), mal um normative Aspekte (Warum entspricht die Zusammensetzung des Bundestages nicht der sozialen Zusammensetzung der Bevölkerung?). Manchen Fragen merkt man dabei an, dass sie im Wissen um die Antwort formuliert wurden, die Antwort diktiert also die Frage wie bei: Wofür erhielt Kurt Schumacher den ersten Sitzungsausschluss im Bundestag?, oder: Ist das Reichstagsgebäude geschrumpft?

Feldkamp greift für diese Publikation auf seine bisherigen Veröffentlichungen zu Daten des Bundestages, zum Amt des Bundestagspräsidenten, zum Frack (Warum tragen die Saaldiener einen Frack?) und zum Hammelsprung (Woher hat der „Hammelsprung“ seinen Namen?) zurück. So finden sich nebeneinander viele nützliche Daten, die anderswo schon gedruckt wurden, Anekdotenhaftes und Listen, die für ein Alltagsgespräch nützlich sein können (häufigste Vornamen der Bundestagsabgeordneten oder auf immerhin elf Seiten Beispiele für Ordnungsrufe).

Der Band hinterlässt einen zwiespältigen Eindruck. Er enthält einerseits viele Informationen in kompakter Form, die auch den schon Wissenden bereichern können (zum Beispiel protokollarische Rangordnung im Bundestag). Es kann auch hilfreich sein, die Daten wie hier schnell griffbereit in einem Taschenbuch zu haben. Andererseits wirkt das Buch wie ein 
Sammelsurium ähnlich den in den letzten Jahren populär gewordenen Listenwerken à la Schott. Feldkamps Anspruch, es könne ein Wegbegleiter durch den Bundestag sein (S. 5), wird so nicht eingelöst. Hinzu kommt, dass es einige Ungenauigkeiten gibt: So zählt die Reichstagskuppel seit 2000 pro Jahr zwischen 585.605 und 744.463 Besucher, die bei Feldkamp im Fließtext zu fast einer Million pro Jahr werden (S. 198). Es werden außerdem keine Quellen angegeben, was bei der Fülle an statistischen Informationen wünschenswert gewesen wäre. Es hätte zum Beispiel interessiert, woher die Zahlen über die Größe von Parlamentsbibliotheken in Europa stammen (S. 202). Schließlich ist die Gliederung nicht stimmig. Abgeordnete werden beispielsweise in drei Abschnitten behandelt (Die Mitglieder des Bundestages; Aus dem Arbeitsalltag eines Abgeordneten; Haushalt und Diäten).

Die Veröffentlichung hätte mehr systematische Auswahl und Recherche verdient gehabt. Der Ansatz, weit verstreutes Wissen zu bündeln, ist lobenswert; doch er überzeugt in dieser Umsetzung nicht vollständig. Vielleicht führt auch der Titel in die Irre, der Hoffnung auf einen knappen und systematischen Wegweiser durch den Bundestag weckt. Zudem verwundert, dass aus den angekündigten 100 Fragen und Antworten tatsächlich 121 werden.

Beide Publikationen führen somit auf zwei unterschiedlichen Wegen an den Bundestag heran. Auf eine Monographie für eine größere Leserschaft zu Deutschlands Parlament, die sich auch der Dilemmata im Parlamentsverständnis annimmt ${ }^{4}$, muss allerdings weiter gewartet werden.

Franziska Höpcke

4 Vgl. Gerhard Loewenberg, Paradoxien des Parlamentarismus. Historische und aktuelle Gründe für Fehlverständnisse in Wissenschaft und Öffentlichkeit, in: ZParl, 38. Jg. (2007), H. 4, S. 816 -827 .

\section{Lesenswertes Brevier zur parlamentarischen Symbolik}

Feldkamp, Michael F. und Dirk Kunze: Mit Frack im Parlament. Ein Beitrag zur parlamentarischen Kultur und politischen Symbolik im Deutschen Bundestag, Nomos Verlagsgesellschaft, Baden-Baden 2009, 79 Seiten, € 19,90.

Das parlamentarische Protokoll der Bundesrepublik verzichtet auf übermäßigen Pomp und ausladende Gesten der Macht. Der Bundestag versteht sich als Arbeitsparlament, und dieses Selbstverständnis als geräuschlos arbeitender Gesetzgeber soll auch in der staatstragenden Symbolik zum Ausdruck kommen. Auch nach dem Umzug des Parlaments in das geschichtsgesättigte Reichstagsgebäude triumphiert im Plenarsaal die kühle Routine sachorientierter Parlamentsarbeit über aufwendiges Staatstheater. Trotz dieser Verzichtsästhetik hat der Parlamentsbetrieb der Bundesrepublik seine eigene Formensprache und Darstellungsformen der repräsentativen Demokratie entwickelt. Dazu gehören unter anderem die Saaldiener des Bundestages mit ihrem Frack, die zu einem „unverwechselbare[n] Bestandteil des parlamentarischen Alltags im Bundestag und damit zu einem Symbol der parlamentarisch-repräsentativen Demokratie in der Bundesrepublik avanciert“" sind (S. 9). 